\title{
Excitation of Alfvén impulse by the anomalous resistance onset on the auroral field lines
}

\author{
V. Pilipenko ${ }^{1}$, N. Mazur ${ }^{1}$, E. Fedorov ${ }^{1}$, T. Uozumi ${ }^{2}$, and K. Yumoto ${ }^{2}$ \\ ${ }^{1}$ Institute of the Physics of the Earth, Moscow, Russia \\ ${ }^{2}$ Space Environment Research Center, Kyushu University, Fukuoka, Japan
}

Received: 11 September 2004 - Revised: 8 March 2005 - Accepted: 29 March 2005 - Published: 3 June 2005

\begin{abstract}
The onset of anomalous resistance in a layer on auroral field lines is shown to be accompanied by the excitation of an Alfvénic impulse (AI). The generated AI marks the transition of the global magnetosphere-ionosphere instability into an explosive phase with positive feedback. The spatial structure of this impulse both in space and on the ground has been described analytically and numerically under the thin layer approximation. The field-aligned currents transported by the Alfvénic impulse are concentrated near the edges of the layer with anomalous resistivity, whereas the reverse currents are spread throughout the layer. For some parameters of the layer the ionospheric attenuation of even small-scale structures is not dramatically large, so the magnetic response to the generated AI may be observed on the ground. The considered event on 3 January 1997 with magnetic-auroral intensification observed by ground magnetometers and Polar UV imager agrees with the model proposed.
\end{abstract}

Keywords. Magnetospheric physics (MHD waves and instabilities; magnetosphere-ionosphere interactions; auroral phenomena)

\section{Introduction}

Though Pi2 pulsations belong to the most well-known types of ULF waves, still there is no confirmative physical interpretation of their generation mechanism. To some extent, this situation is quite natural because it is unrealistic to develop a final theory of Pi2 before the build-up of a general theory of substorms. Nowadays, the substorm theory is still an unresolved problem of geophysics. However, as seismic waves provide information about earthquakes, though the physics of earthquakes is not fully understood, in a similar way the Pi2 waves may be used as a tool for the understanding and monitoring of the substorm process.

Correspondence to: V. Pilipenko

(pilipenk@augsburg.edu)
A typical Pi2 waveform-damping short train implies that $\mathrm{Pi} 2$ is a transient response to some rapid large-scale change of the night-side magnetospheric current system (Baumjohann and Glassmeier, 1984; Olson, 1999). Because of multiple rapid plasma processes in the nightside magnetosphere, it should be expected that Pi2-like transient disturbances can be triggered by several possible magnetospheric drivers. General association within a few minutes was established between the Pi2 waves and the substorm onset, auroral breakup, onset of geomagnetic bay, and explosive phenomena in the nightside magnetosphere, such as the cross-tail current disruption, dipolarization, X-line formation, bursty bulk flows, etc. (Shiokawa et al., 1998; Kepko and Kivelson, 1999; Liou et al., 1999, 2000; Kepko et al., 2001). Moreover, the existence of several onset mechanisms or their synthetic combinations is possible (Lui, 1996). The growth phase of a substorm is often accompanied by a series of localized auroral activations, from small-scale arc activation to pseudobreakup (Shiokawa et al., 2002). Probably only one from this series of activations is the "main onset", leading to the explosive substorm development. Moreover, transient magnetic signals can be excited in the ionosphere by sudden changes in the ionospheric conductance under the impact of precipitating electrons (Maltsev et al., 1974), or by the switch-on of the energetic particles source (Trakhtengertz and Feldstein, 1988). Thus, to identify a specific mechanism of the substorm global instability, more detailed timing and examination of the onset fine structure are necessary.

Here we draw attention to the possibility of an additional mechanism of Pi2 generation related to the onset of an anomalous resistance on auroral field lines. The sudden onset of field-aligned resistance is to be accompanied by the excitation of Alfvénic impulse (AI), as was originally suggested by Arykov and Maltsev (1983). We develop a mathematical formalism for the description of the AI spatial structure and discuss some implications of this model. Also, we provide some observational evidence in favor of this hypothesis. We believe that the proposed mechanism might be an important element of the substorm-Pi2 relationship. 


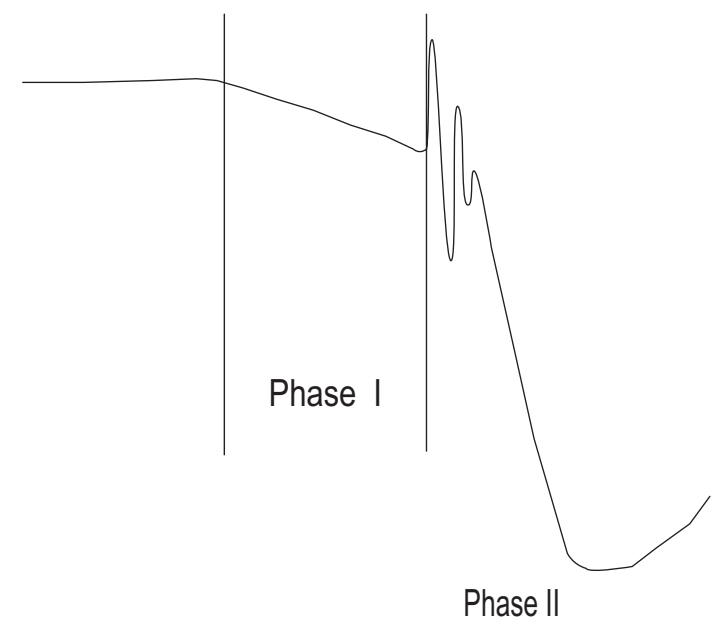

Fig. 1. A schematic scenario of the temporal two-stage evolution of the substorm explosive phase.

\section{General physics of the magnetosphere-ionosphere in- teraction}

First, we outline the role of anomalous resistance in the magnetosphere-ionosphere interaction. The origin of a substorm is related to the reconfiguration of global currents in the nightside magnetosphere. The key element of this reconfiguration is the disruption of the cross-tail current and its divergence into field-aligned currents towards or away from the ionosphere. This process may be visualized as a sporadic electric discharge of the magnetospheric cross-tail potential through the ionosphere, though the specific mechanism of this global instability has not been firmly identified yet. In the region of upward field-aligned current $j_{0}$ the emergence of an anomalous resistivity layer (ARL) with a finite field-aligned conductivity $\sigma_{\|}$results in the emergence of an anomalous electric field $E_{\|} \simeq j_{0} / \sigma_{\|}$. This field-aligned $E_{\|}$ accelerates down-going electrons that precipitate in the ionosphere. The accelerated electrons, in turn, cause additional ionization of the ionosphere and activation of the auroral activity. The ionospheric ionization and relevant modification of the ionospheric conductance make feasible various mechanisms of feedback in the coupled ionosphere-magnetosphere system (Lysak and Dum, 1983; Pokhotelov et al., 2001; Lysak and Song, 2002). In a general sense, it may be speculated that the cause of substorm is some global instability of the ionosphere-magnetosphere system. After the turn-on of the positive feedback in the system this instability transforms itself into an explosive phase with a much higher, possibly non-linear, growth rate.

At the same time the sudden "switch-on" of the anomalous resistivity results in the excitation of AI. Details of the excitation mechanism and peculiar spatial structure of this impulse will be considered in this paper. According to the proposed scenario, the AI occurrence signifies the switch-on of $\sigma_{\|}$along an auroral field line and thus is the indicator of the transition of a global magnetospheric instability into the ionosphere-coupled phase with a much higher growth rate.

The occurrence of resonant features of the ionospheremagnetosphere system, such as the magnetospheric Alfvén resonator (with typical eigenfrequencies at auroral latitudes $f \sim 10 \mathrm{mHz})$, ionospheric Alfvén resonator $(f \sim$ few $\mathrm{Hz})$ (Belyaev et al., 1990), and the resonator formed between the ionosphere and the auroral acceleration region $(f \sim$ few tenths of Hz) (Pilipenko et al., 2002) can produce an oscillatory transient response to the excited AI.

Thus, the temporal evolution of a substorm onset can be visualized as a sequence of the following phases, as schematically illustrated in Fig. 1:

I. Growth of the field-aligned current $j_{0}$ due to energy disbalance in the nightside magnetosphere. At this stage there is still no feedback between the magnetosphere and ionosphere. This stage could be revealed from ground magnetograms as a gradual slow decrease of the $\mathrm{H}$ component of the geomagnetic field, and can be coined as a "substorm precursor" (Groot-Hedlin and Rostoker, 1987). At some moment, when $j_{0}$ exceeds a plasma instability threshold the sudden (on MHD time scale) onset of anomalous $\sigma_{\|}$occurs. This moment, as suggested by Arykov and Maltsev (1983), is to be accompanied by the appearance of transient AI.

II. Acceleration of precipitating electrons by anomalous $E_{\|}$and modification of the ionospheric conductance, thus establishes a feedback between the ionosphere and the magnetosphere.

From the scenario outlined above it follows that the transition from phase I to phase II actually means a transfer of the global magnetospheric instability into an explosive phase (detonation) with a positive ionospheric feedback.

\section{MHD model of a thin ARL}

Here we develop a mathematical formalism for the description of the AI generation during the switch-on of anomalous resistivity on auroral field lines. We adapt the ARL model, schematically shown in Fig. 2, where the straight geomagnetic field is directed vertically up, $\mathbf{B}_{0}=B_{0} \hat{\mathbf{z}}$. The homogeneous magnetospheric plasma has zero transverse static conductivity and infinite field-aligned conductivity $\sigma_{\|}=\infty$. Only at some altitude, which is considered to be the origin of the field-aligned coordinate $z$, the finite $\sigma_{\|}$occurs inside the ARL with the thickness $b$ and width $2 a$.

The considered mathematical model is based on the system of Maxwell's equations

$\nabla \times \mathbf{E}=-\frac{1}{c} \partial_{t} \mathbf{B}, \quad \nabla \times \mathbf{B}=\frac{4 \pi}{c} \mathbf{j}$,

augmented by Ohm's law

$\mathbf{j}_{\perp}=\Sigma_{A} V_{A}^{-1} \partial_{t} \mathbf{E}_{\perp}, \quad j_{z}=\sigma_{\|} E_{z}$,

where $\Sigma_{A}=c^{2} /\left(4 \pi V_{A}\right)$ is the Alfvén wave conductance. 
In this system the possible MHD disturbances are described by the following decoupled equations for Alfvén waves, carrying the field-aligned current $j_{z}$, and compressional waves, carrying the field-aligned magnetic field disturbance $B_{z}$ :

$\partial_{t t} j_{z}-V_{A}^{2} \partial_{z z} j_{z}=\frac{c^{2}}{4 \pi} \nabla_{\perp}^{2} \partial_{t}\left(\sigma_{\|}^{-1} j_{z}\right)$,

$\partial_{t t} B_{z}-V_{A}^{2} \nabla^{2} B_{z}=0$.

We assume that the ARL is a thin layer as compared to the Alfvén wave length. Therefore, the thin layer approximation can be used, that is the ARL thickness $b \rightarrow 0$, whereas its total resistance $Q(x, y, t)=b / \sigma_{\|}$remains finite.

In this approximation the simple Alfvén wave equation is valid in the upper $(z>0)$ and lower $(z<0)$ hemi-spaces

$\partial_{t t} j_{z}-V_{A}^{2} \partial_{z z} j_{z}=0$.

This equation must be supplemented with two boundary conditions at the interface $z=0$ between two hemi-spaces, separated by a thin layer $(b \rightarrow 0)$ with the resistivity $Q$. The first condition is the requirement of the continuity of field-aligned current $j_{z}(x, y, z, t)$ across the ARL, which enables us to consider the function $j_{z}^{(0)}(x, y, t)=j_{z}(x, y, 0, t)$ (the superscript (0) indicates that the current is considered inside the ARL). The second boundary condition is obtained by integration of the first equation from the system (3) across the layer and subsequent transition to the limit $b \rightarrow 0$, as follows

$\left\{\partial_{z} j_{z}\right\}_{z=0}+\nabla_{\perp}^{2} \partial_{t}\left[R(x, y, t) j_{z}^{(0)}\right]=0$.

Here $\quad R(x, y, t)=\frac{c^{2}}{4 \pi V_{A}^{2}} Q=\Sigma_{A} Q V_{A}^{-1} \quad$ is the normalized resistance of the ARL, and $\left\{\partial_{z} j_{z}\right\}_{z=0}=\partial_{z} j_{z}(x, y,+0, t)-\partial_{z} j_{z}(x, y,-0, t) \quad$ is the jump in the current density derivative across the ARL.

\section{Generation of Alfvénic impulse during the switch-on of anomalous conductivity}

Let us suppose that at $t<0$ a steady homogeneous current $j_{z}(x, y, z, t)=j_{0}$ flows along the field lines with zero resistance $Q=0$. Then, at $t \geq 0$ anomalous resistivity is turned on, described by some function $Q(x, y)>0$ at the plane $z=0$ (Fig. 2). The onset of $\sigma_{\|}$induces the disturbance of fieldaligned current inside the ARL, which is characterized by the current density $j_{z A}(x, y, t)=j_{z}^{(0)}(x, y, t)-j_{0}$. The solution of Eq. (4) for $t \geq 0$ for the whole space is determined by the function $j_{z A}(x, y, t)$, defined at the border $z=0$. This solution evidently has the form of outward propagating disturbances:

$j_{z}(x, y, z, t)=j_{0}+j_{z A}\left(x, y, t-z / V_{A}\right), \quad z \geq 0 ;$
$j_{z}(x, y, z, t)=j_{0}+j_{z A}\left(x, y, t+z / V_{A}\right), \quad z<0$.

The second boundary condition (5) enables us to obtain an equation to determine the induced field-aligned current density $j_{z A}(x, y, t)$. Substituting the jump in the current density derivative across the ARL found from Eq. (6),

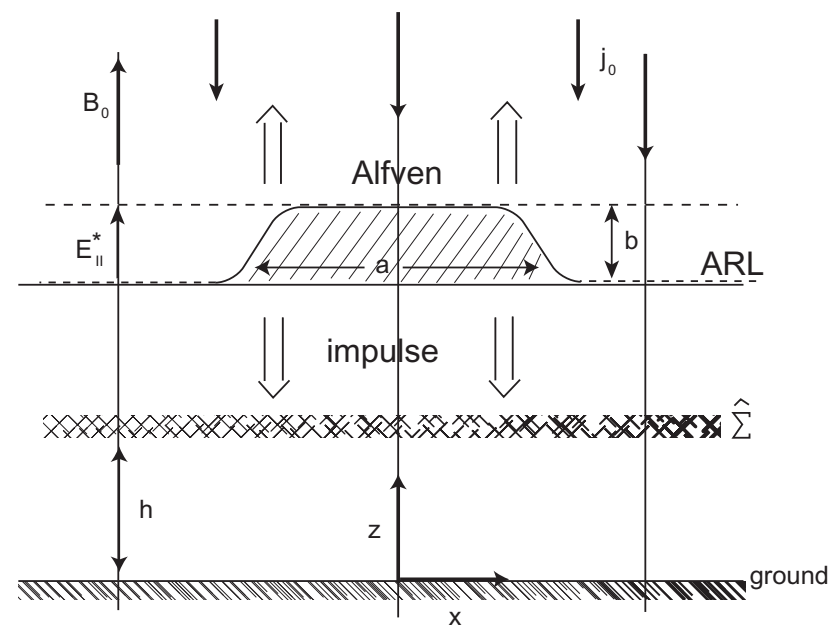

Fig. 2. Model of the Alfvén impulse generation by the ARL with anomalous electric field $E_{\|}$.

$\left\{\partial_{z} j_{z}\right\}_{z=0}=-2 V_{A}^{-1} \partial_{t} j_{z A}$, into the condition (5) one obtains the equation for the induced current as follows:

$\partial_{t}\left\{-2 j_{z A}+V_{A} \nabla_{\perp}^{2}\left[R\left(j_{0}+j_{z A}\right)\right]\right\}=0$.

This relationship means that the expression in curly brackets does not depend on time. Since at $t<0$ the current disturbance $j_{z A}=0$ and resistance $R=0$, the above equation reduces to the following

$$
-2 j_{z A}+V_{A} \nabla_{\perp}^{2}\left[R\left(j_{0}+j_{z A}\right)\right]=0 .
$$

The equation obtained yields the relationship between the induced current $j_{z A}(x, y, t)$ of AI and external current $j_{0}$. This equation may be re-written as a relationship between the current inside the ARL and the external background current

$j_{z}^{(0)}-\frac{V_{A}}{2} \nabla_{\perp}^{2}\left(R j_{z}^{(0)}\right)=j_{0}$.

From relationship (7) the equation from (Arykov and Maltsev, 1983) for the wave potential $\varphi$ (bound to the transverse electric field by the relation $\left.\mathbf{E}_{\perp}=-\nabla_{\perp} \varphi\right)$ can be obtained by the substitution $j_{z}^{(0)}=-2 Q^{-1} \varphi=-2 \Sigma_{A}\left(V_{A} R\right)^{-1} \varphi$ as follows

$$
-2 Q^{-1} \varphi+\Sigma_{A} \nabla_{\perp}^{2} \varphi=j_{0} .
$$

Note that the potential $\varphi$ (as well as $\mathbf{E}_{\perp}$ ) is discontinuous across the layer, and Eq. (8) corresponds to the potential at the upper ARL boundary, i.e. $\varphi=\varphi(x, y,+0, t)$. Due to the system symmetry $\varphi(z=+0)=-\varphi(z=-0)$, so the potential drop across the layer is $\{\varphi\}=-2 \varphi$.

The induced field-aligned current density $j_{z A}(x, y, t)$ can be expressed directly through the potential $\varphi$ (Arykov and Maltsev, 1983). From the linearized basic Eqs. (1) and (2) one obtains the relation

$\partial_{z} j_{z}=-\frac{c^{2}}{4 \pi V_{A}^{2}} \partial_{t}\left(\nabla \cdot \mathbf{E}_{\perp}\right)$ 


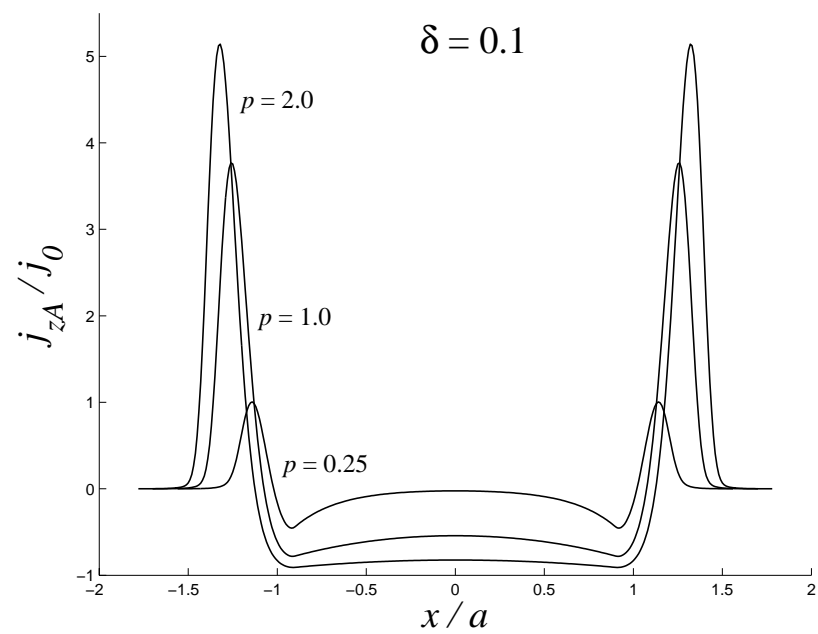

Fig. 3. The spatial structure of the current $j_{z A}(x)$ for a fixed spatial scale $a$ and smoothness parameter $\delta=0.1$, for several values of $p=\lambda_{A 0} / a$.

From Eq. (9) one may obtain the relationship for the propagating Alfvén waves (6) as follows:

$j_{z A}= \pm \Sigma_{A}\left(\nabla \cdot \mathbf{E}_{\perp}\right)=\mp \Sigma_{A} \nabla_{\perp}^{2} \varphi$,

where the upper/lower signs correspond to the upper/lower ARL boundary (because of the symmetric jump in the potential across the layer).

The interaction of AI with the ARL can be categorized by the Alfvén damping scale $\lambda_{A}=\sqrt{\Sigma_{A} Q / 2}$, introduced by Vogt and (1998), and Fedorov et al. (2001). This parameter is the scale of the flux tube where the field-aligned conductance matches the Alfvén wave conductance. As the resistance $Q$ of the ARL increases the parameter $\lambda_{A}$ grows larger.

\section{Transverse spatial structure of Alfvén impulse}

We assume that the system under consideration is infinitely extended along the $y$ axis, similar to the auroral arc configuration. Equation (8) for the AI potential in a 1-D inhomogeneous (along $x$ axis) plasma is as follows:

$\varphi^{\prime \prime}-\kappa(x)^{2} \varphi=j_{0} / \Sigma_{A}$,

where $\kappa(x)^{2}=\lambda_{A}^{-2}(x)=2 / \Sigma_{A} Q(x)$. For the profile of $Q(x)$ with maximal value $Q_{0}$ and transverse scale $a$ (Fig. 2) this equation can be re-written as:

$\varphi^{\prime \prime}-\frac{\kappa_{0}^{2}}{g(x / a)} \varphi=\frac{j_{0}}{\Sigma_{A}}$,

where $\kappa_{0}=\lambda_{A 0}^{-1}=\left(2 / \Sigma_{A} Q_{0}\right)^{1 / 2}$. The function $g(x / a)=Q(x) / Q_{0}(0<g \leq 1)$ describes the transverse spatial structure of the ARL. Equation (11) can be normalized using the dimensionless variable $\xi=x / a$ and the dimensionless potential $u=\varphi / \varphi_{0}$, where $\varphi_{0}=j_{0} Q_{0} / 2=j_{0} /\left(\Sigma_{A} \kappa_{0}^{2}\right)$, as follows:

$p^{2} u^{\prime \prime}-u / g(\xi)=1$.

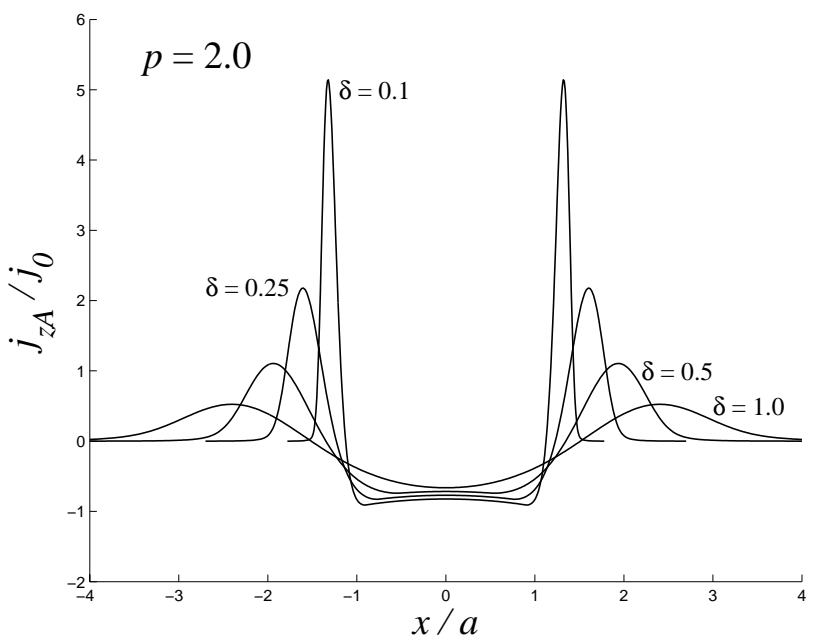

Fig. 4. The structure of induced current $j_{z A}$ for certain fixed scale $a$ and parameter $\lambda_{A 0}(p=2.0)$ with respect to the parameter $\delta$.

Here the parameter $p=\left(\kappa_{0} a\right)^{-1}=\lambda_{A 0} / a$ is the ratio between the characteristic Alfvén damping scale and the ARL width.

The induced electric field $E_{x}$ and the induced field-aligned current density $j_{z A}$ can be derived via the dimensionless potential $u(\xi)$ :

$$
\begin{aligned}
& E_{x}=-\partial_{x} \varphi=-\left(\varphi_{0} / a\right) \partial_{\xi} u=-E_{0} p \partial_{\xi} u, \\
& j_{z A}=-\Sigma_{A} \partial_{x x} \varphi=-j_{0}[1+u / g]=-j_{0} p^{2} \partial_{\xi \xi} u .
\end{aligned}
$$

Here $E_{0}=\varphi_{0} \kappa_{0}=j_{0} \lambda_{A 0} / \Sigma_{A}$ denotes the characteristic value of $E_{x}$.

Let us suppose that the transverse profile of the ARL is described by an even function $g(\xi)$, determined by the formulas:

$g(\xi)=\left\{\begin{array}{lll}1 & \text { at } & |\xi| \leq 1-\delta \\ \cosh ^{-2}[1+(|\xi|-1) / \delta] & \text { at } & |\xi| \geq 1-\delta .\end{array}\right.$

The parameter $\delta(0<\delta \leq 1)$ in Eq. (14) characterizes the smoothness of the ARL edges. Thus, when $\delta=1$ the profile is smooth, namely $g(\xi)=(\cosh \xi)^{-2}$, whereas when $\delta \rightarrow 0$ the distribution tends to be step-wise (considered in the next section).

Equation (12) has been solved numerically as a boundary problem with a zero boundary condition at infinity for the chosen ARL profile (14). Figure 3 shows the calculation results for the spatial structure of the current $j_{z A}(x)$ for a fixed spatial scale $a$ and smoothness parameter $\delta=0.1$ for several values of the ARL resistance characterized by the ratio $p=\lambda_{A 0} / a$. The induced current is concentrated at the ARL edges. Inside the ARL, a more widely distributed reverse induced current flows which tends to compensate the background external current. The larger the resistance of the ARL, the bigger the amplitude will be of the excited current impulse $j_{z A}$. For example, $j_{z A} / j_{0} \simeq 5$ for $p=2.0$, but it drops to $j_{z A} / j_{0} \simeq 1$ for $p=0.25$.

Figure 4 shows the structure of induced $j_{z A}$ for a certain fixed scale $a$ and parameter $\lambda_{A 0}$ with respect to the 


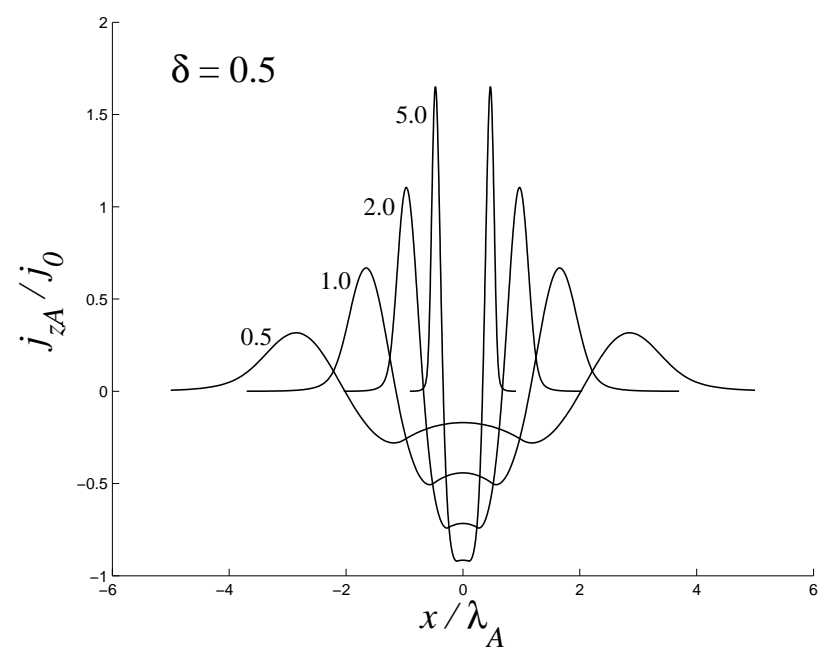

Fig. 5. The spatial structure of induced current $j_{z A}(x)$ for a fixed characteristic length $\lambda_{A 0}$ with respect to the ARL width characterized by $p$ (indicated near curves).

smoothness of the ARL edges characterized by parameter $\delta$. Comparison of curves for various $\delta$ shows that the sharper the ARL edges (the smaller $\delta$ ), the larger the peak current density will be for the same ARL resistance (the same $p$ ). The peak current density can even exceed several times the density of the original current $j_{0}$, when $\delta \ll 1$.

Figure 5 shows the calculated spatial structure (in dimensionless variable $\left.x / \lambda_{A 0}\right)$ of the induced current under the fixed Alfvén scale $\lambda_{A 0}$ and ARL smoothness $\delta=0.5$, for several values of $p$ (i.e. for different scales $a$ ). It follows from this plot that for the same magnetospheric parameters the peak intensity of the induced current increases with a decrease of the ARL width (increase of $p$ ).

However, the integral induced current flowing into the ionosphere depends on the ARL scale in a different way as compared to the current density $j_{z A}$. The occurrence of the ARL causes the redistribution of the background external current in such a way that the total disturbed current vanishes, namely

$$
\int_{j_{z A}>0} j_{z A}(x) d x=-\int_{j_{z A}<0} j_{z A}(x) d x .
$$

The total current integrated over the regions with a positive current density actually is determined by the peak values of the induced electric field $E_{x}(x)$ in the ARL. Indeed, because of the relation (10) and owing to the assumed symmetry of the model (so $\max E_{x}=-\min E_{x}$ ), the total current is related to max $E_{x}$ as follows:

$J_{z A}=\int_{j_{z A}>0} j_{z A}(x) d x=2 \Sigma_{A} \max E_{x}$.

Figure 6 shows the structure of $E_{x}(x)$ under fixed $\lambda_{A 0}$ and $\delta=0.5$ for various scales $a$. Each curve is denoted by the relevant value of $p=\lambda_{A 0} / a$. The insert in Fig. 6 shows the

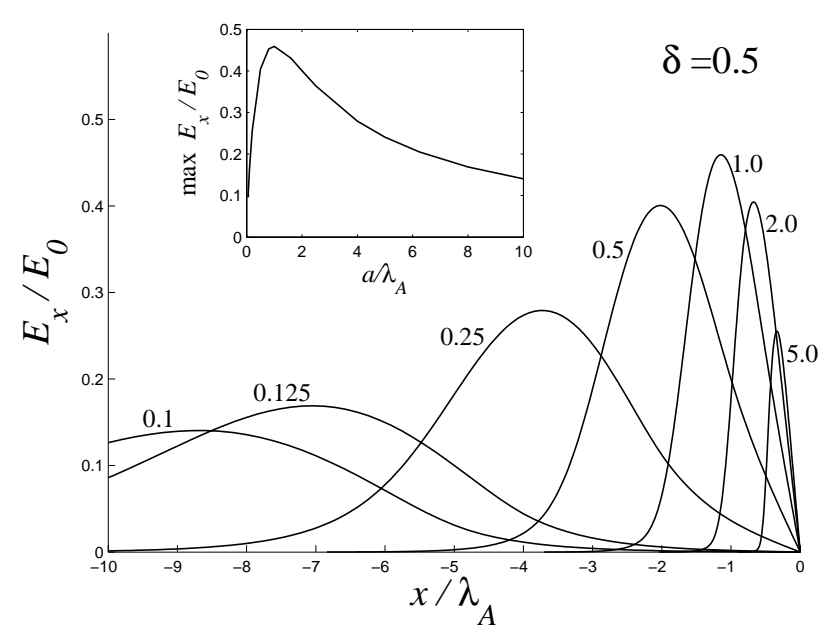

Fig. 6. The electric field along the ARL and the dependence of $\max E_{x}$ on the scale $a$.

dependence of $\max E_{x}$ in respect to $a$. As it can be seen from this figure, the integrated current $J_{z A} \propto \max E_{x}$ grows $(\propto a)$ under $a \ll \lambda_{A 0}$, and gradually decreases at large $a$. The maximal disturbance of total current is produced by the ARL with scales $a$ comparable to the Alfvén damping scale, that is $a / \lambda_{A 0} \sim 1$.

\section{Structure of the Alfvén impulse in a step-wise ARL}

In a simplified case, corresponding to the elongated box-like profile of the ARL $(\delta \rightarrow 0)$, the AI spatial structure can be described analytically (Arykov and Maltsev, 1983). From Eq. (11) with $g(\xi)=\eta(1-|\xi|)$, where $\eta(\xi)$ is the Heaviside's function $(\eta(\xi<0)=0$ and $\eta(\xi \geq 0)=1)$, the expression for potential follows

$\varphi=\varphi_{0} \frac{\cosh \left(\kappa_{0} x\right)-\cosh \left(\kappa_{0} a\right)}{\cosh \left(\kappa_{0} a\right)} \eta(a-|x|)$.

The electric field in the ARL is

$E_{x}=-\frac{\partial \varphi}{\partial x}=E_{0} \frac{\sinh \left(\kappa_{0} x\right)}{\cosh \left(\kappa_{0} a\right)} \eta(a-|x|)$.

The peak values of electric field are reached at the edges $\quad x= \pm a: \quad E_{x}^{(m)}=\left|E_{x}( \pm)\right|=E_{0} \tanh \left(\kappa_{0} a\right)$. For small-scale structures, $a \ll \lambda_{A 0}$, the peak value is $E_{x}^{(m)} \simeq E_{0} \kappa_{0} a=j_{0} a / \Sigma_{A}$.

Disturbance of current is

$$
\begin{aligned}
j_{z A}= & \Sigma_{A} \frac{\partial E_{x}}{\partial x}=-j_{0} \frac{\cosh \left(\kappa_{0} x\right)}{\cosh \left(\kappa_{0} a\right)} \eta(a-|x|) \\
& +\frac{j_{0}}{\kappa_{0}} \tanh \left(\kappa_{0} a\right)[\delta(x+a)+\delta(x-a)],
\end{aligned}
$$

where $\delta(\xi)$ is the $\delta$-function. The second term corresponds to the infinitely thin localized current at the ARL edges and the first term corresponds to the reverse current distributed 


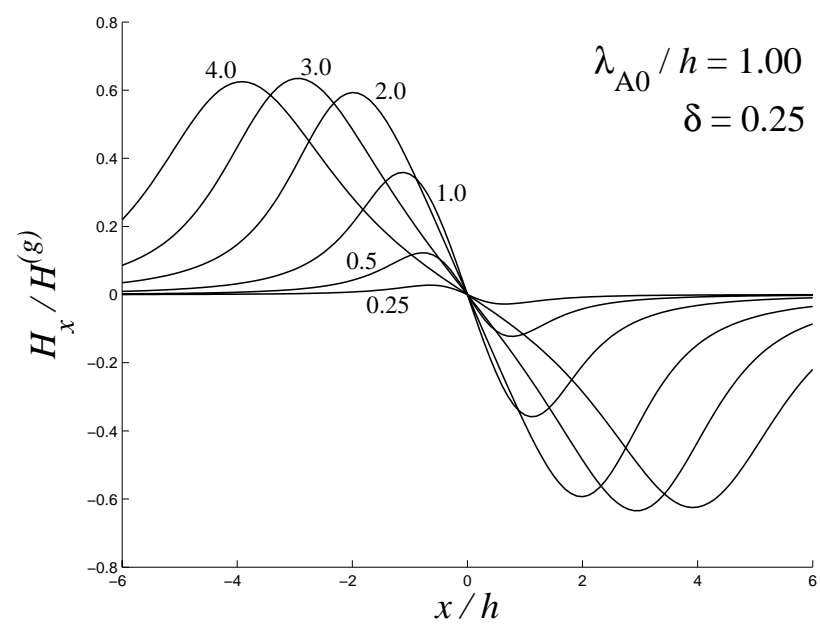

Fig. 7. The numerically calculated spatial structure of the ground magnetic response for several values of $\alpha$ (indicated near curves) and $\lambda_{A 0} / h=1.0$.

within the ARL. The maximal value of the current disturbance, $j_{0}$ at $x= \pm a$, compensates for the background current. In the case $a \gg \lambda_{A 0}$, the region where the reverse current is significant $\left(j_{z A} \simeq j_{0}\right)$ is very narrow as compared to the ARL width $a$, whereas at $a \ll \lambda_{A 0}$, the reverse current $j_{z A} \simeq j_{0}$ occurs throughout the entire ARL. The integrated current $J_{z A} \propto \max E_{x}=E_{0} \tanh \left(a / \lambda_{A 0}\right)$ grows in a monotonic way upon the increase of scale $a$, approaching the asymptotic value $2 \Sigma_{A} E_{0}$.

\section{Ground effect of the Alfvén impulse}

The ground effect of the 1-D AI-related current $j_{z A}(x, t)$ is produced by the ionospheric Hall current along the $y$ axis. This current is induced by the $E_{x}$ component of the incident AI:

$j_{H}=\frac{2 \Sigma_{H} \Sigma_{A}}{\Sigma_{A}+\Sigma_{P}} E_{x}$

Expression (15) is obtained using the boundary conditions upon reflection of an Alfvén wave from the thin ionosphere with the height-integrated conductivities $\Sigma_{H}$ and $\Sigma_{P}$.

The ground magnetic effect of the ionospheric Hall current (15) can be found using Biot-Savart's law. Near the surface of the highly-conductive ground the $H_{z}$ component vanishes and the $H_{x}$ component doubles, so

$H_{x}(x)=\frac{4 h}{c} \int_{-\infty}^{\infty} \frac{j_{H}\left(x^{\prime}\right) d x^{\prime}}{h^{2}+\left(x^{\prime}-x\right)^{2}}$,

where $h$ is the altitude of the conductive current above the Earth's surface. The sign in Eq. (16) corresponds to the Northern Hemisphere.

In order to apply directly the estimates of the ground effect of the solution for the dimensionless potential $u(\xi ; p)$ of
Eq. (12), relationship (16) has been normalized by the scale $a$. Introducing the parameter $\alpha=a / h$, that is the ratio between the ARL width and the height of the ionosphere, this relationship can be presented as:

$H_{x}(\xi ; \alpha, p)=-H^{(g)}\left(\frac{\lambda_{A 0}}{h}\right)^{2} \int_{-\infty}^{\infty} \frac{\partial_{\xi^{\prime}} u\left(\xi^{\prime} ; p\right) d \xi^{\prime}}{1+\alpha^{2}\left(\xi^{\prime}-\xi\right)^{2}}$.

Here we have introduced a characteristic value of the ground magnetic field $H^{(g)}=8 \Sigma_{H}\left(\Sigma_{A}+\Sigma_{P}\right)^{-1} j_{0} h c^{-1}$ that does not depend on the parameters $\lambda_{A 0}$ and $a$ and refers to the case when the three considered linear scales, $\lambda_{A 0}, a$, and $h$, are the same.

The spatial ground structure of the normalized magnetic disturbance $H_{x}(x) / H^{(g)}$ has been calculated numerically and it is shown for $\delta=0.25$ in Fig. 7 for several values of $\alpha$ and parameter $\lambda_{A 0} / h=1.0$. The ground magnetic signal has a bi-polar structure: it changes polarity beneath the ARL.

Geometrical attenuation of magnetic response upon transmission through the ionosphere is weak for large-scale structures when $\alpha \geq 1$ or $a \geq h$. In this case the peak magnetic values $H_{x}^{(m)}=\max \left|H_{x}\right| \simeq H^{(g)}$. For example, in the case $\lambda_{A 0} / h=1.0$ at $\alpha=3.0$ the peak value of the ground magnetic disturbance is $H_{x}^{(m)} \simeq 0.63 H^{(g)}$ (Fig. 7). The attenuation of the ground magnetic response from small-scale structures $a \ll h$ becomes significant. For example, for the same plasma parameters, $H_{x}^{(m)} \simeq 0.36 H^{(g)}$ for $\alpha=1.0$, and $H_{x}^{(m)} \simeq 0.003 H^{(g)}$ for $\alpha=0.1$.

Let us examine the attenuation of small-scale structures in greater detail. In the case of small-scale ARL as compared with the height of the ionosphere, i.e. $\alpha \ll 1$, relationship (17) can be presented as the 2-D dipole approximation, namely

$H_{x}(x)=16 M \frac{h x}{\left(h^{2}+x^{2}\right)^{2}}$.

Here $M$ is the magnetic moment per unit length along the $y$ axis, created by the Hall current $j_{H}$, namely

$M=\frac{1}{2 c} \int_{-\infty}^{\infty} j_{H}(x) x d x$.

The maximum magnetic disturbance on the ground is achieved at the distance $x^{(m)}$ from the center of the ARL:

$H_{x}^{(m)} \simeq 3 \sqrt{3}|M| h^{-2} \quad$ at $\quad x^{(m)}= \pm h / \sqrt{3}$.

The magnetic moment $M$ may be expressed via the normalized potential $u(\xi ; p)<0$ as follows:

$M=-\frac{1}{8} \lambda_{A 0}^{2} H^{(g)} \alpha U(p), \quad U(p)=-\int_{-\infty}^{\infty} u(\xi ; p) d \xi$

On the order of magnitude, $U \simeq \max |u|$, because the integrand function $u(\xi)$ is exponentially small beyond the unitlength interval.

Now we consider the dependence of the integral function $U(p)$ on $p$, that is the ratio between $\lambda_{A 0}$ and the ARL width 
$a$. It follows from the analysis of the boundary problem for Eq. (12) that when ARL is wide $(p \leq 1)$, the value of $U(p) \simeq 1$. When the ARL is narrow $(p \gg 1)$, the value $U(p)$ becomes as small as $U(p) \sim p^{-} \mu$, with $\mu$ in the interval $0<\mu \leq 2$. The power factor $\mu$ depends on the parameter $\delta$. It is easy to find $\mu$ analytically for the step-wise ARL profile $(\delta \rightarrow 0)$. In this case at $p \gg 1$ the function $u(x ; p) \simeq \frac{1}{2} p^{-2}\left(x^{2}-1\right) \eta(1-|x|)$, and therefore $M(p) \simeq \frac{2}{3} p^{-2}$, that is $\mu=2$. Numerical calculations show that the factor $\mu$ decreases somewhat for more smooth ARL edges. For example, at $\delta=0.05$ it is quite close to the "step-wise" value: $\mu=1.87$, whereas at $\delta=0.25$ this factor $\mu=1.47$.

For a narrow ARL $(\alpha \ll 1)$, two cases are possible depending on the ratio between the characteristic length $\lambda_{A 0}$ and $h$. If $\lambda_{A 0} \ll h$, then

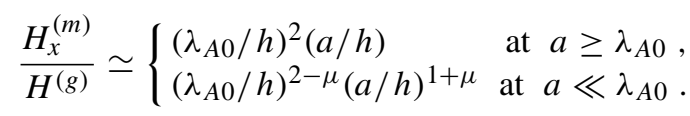

One can see from the above estimate that the maximum magnetic field decays linearly upon the decrease of the scale $a$, and only when $a$ become smaller than $\lambda_{A 0}$ does this decay occur faster, by a power law $a^{1+\mu}$ (e.g. cubic for the stepwise profile).

If $\lambda_{A 0} \geq h$, then under $\alpha \ll 1$ there is only the possible limiting case $a \ll \lambda_{A 0}$, when the peak ground magnetic disturbance $H_{x}^{(m)} / H^{(g)}$ is described by the second relationship from Eq. (20). The above consideration indicates that the ground magnetic disturbance produced by small-scale structures, $\alpha \ll 1$, in some cases decreases not as fast upon the decrease of the ARL scale $a$.

The analytical estimates are confirmed by the numerical calculations of dependence $H^{(m)}(\alpha)$. Figure 8 shows the dependence of normalized peak values of ground magnetic disturbance, $H^{(m)} / H^{(g)}$, with respect to $\alpha$ for several values of $\lambda_{A 0} / h$ (indicated near curves) for the same smoothness parameter $\delta=0.25$ as in Fig. 7. It can be seen that upon the decrease of $\alpha$ the rate of magnetic field decrease is rather slow (nearly linear) in the range of parameters $\lambda_{A 0} / h \leq 0.2$ and $\alpha \geq 0.2$, and faster for $\alpha \leq 0.2$ (power law with factor 2.47). The examination of Fig. 8 shows that the ground magnetic response from small-scale ARL is essentially attenuated. However, this attenuation is not so severe (about an order of magnitude only) in the range of parameters $\alpha \geq 0.2$ and $\lambda_{A 0} / h \geq 0.5$ to prevent the ground observations of the ARL-related AI in some situations.

\section{Model consequences and comparison with observa- tions}

At the initial phase of the reconfiguration of global currents in the nightside magnetosphere during substorm onset, the ionosphere plays a role as only a passive sink of energy for the magnetospheric field-aligned current. Only when this current exceeds a certain threshold does an anomalous resistivity emerge on auroral field lines. In the region of upward

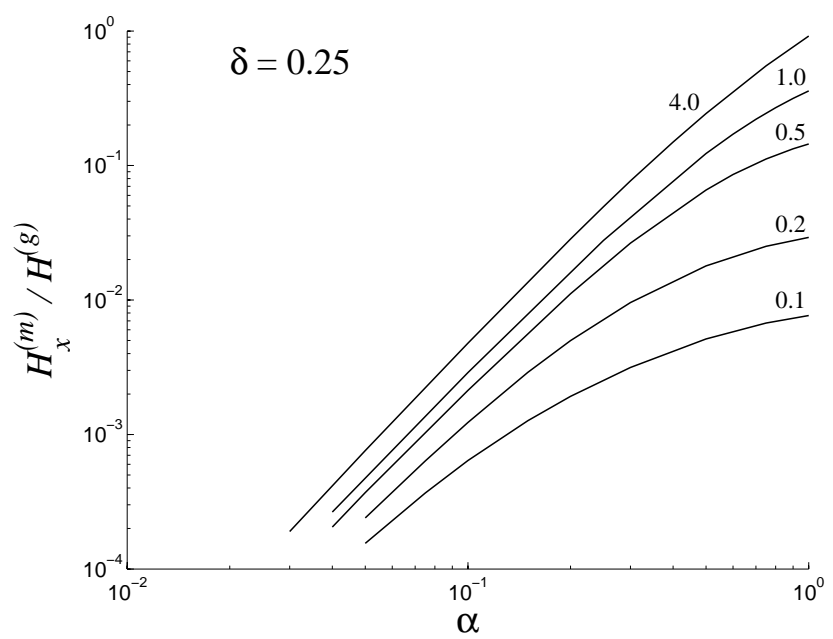

Fig. 8. The numerically calculated dependence of the normalized ground magnetic response peak values on $\alpha$ for several values of $\lambda_{A 0} / h$ (indicated near curves) and $\delta=0.25$.

current, the moment of an anomalous resistance and electric field emergence, as described in Sect. 1, indicates the onset of positive feedback in the magnetosphere-ionosphere system. However, the situations when the considered mechanism can be operative may occur not only during the main onset but during any intensification of field-aligned current of sufficient magnitude and accompanying auroral activation.

Many global observational campaigns (Yumoto et al., 1990; Yeoman et al., 1991; Uozumi et al., 2000, 2004) show that $\mathrm{Pi} 2$ is a global phenomenon which is observed over a wide range of latitudes (until the equatorial latitudes) and LT, even till the dayside (Sutcliffe and Yumoto, 1991). Therefore, a global compressional mode should be involved in the excitation of Pi2. This large-scale compressional mode is probably generated by bursty processes during substorm onset in the nightside magnetosphere. Upon propagation towards the inner magnetosphere this mode illuminates the whole nightside magnetosphere and can be transformed on Alfvén velocity gradients into transient Alfvén oscillations (Baransky et al., 1980). When the field-aligned current density at the front of the disturbance generated by the substorm detonation exceeds the threshold sufficient for the excitation of some high-frequency turbulence (most probably at altitudes about $1 R_{E}$ ), an anomalous resistivity is turned on. This moment is to be accompanied by the excitation of additional local transient AI, as was indicated by Arykov and Maltsev (1983). The theoretic model presented here describes the features of this impulse.

Thus, at auroral latitudes the substorm onset may be accompanied by the superposition of several transient Pi2-like signals. Only at lower latitudes, where the localized auroral disturbances are substantially attenuated does the global part of the disturbance related to compressional mode dominate and can be observed as a clear, isolated Pi2 train. A reliable discrimination of various source mechanisms that contribute 

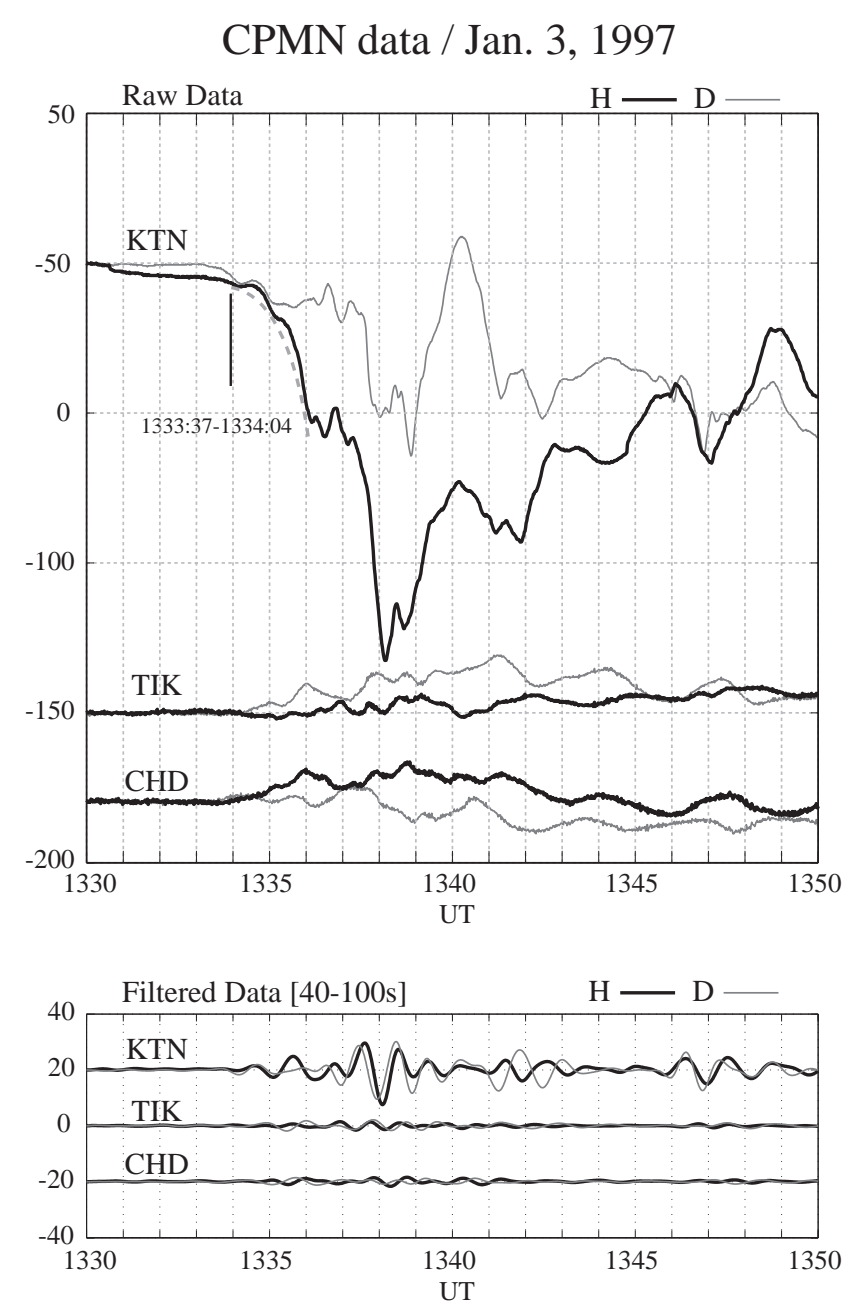

Fig. 9. Magnetic intensification and Pi2 transients, recorded at CPMN stations on 3 January 1997. The vertical lines indicate the moments of the auroral intensifications observed by Polar UV imager.

to the ground ULF disturbance during a substorm break-up can be done with a more dense array of magnetometers with high time resolution. Special methods of analysis are necessary to discriminate contributions from various mechanisms into Pi2 signature. For example, to reveal a fine temporal structure of Pi2 trains, the phase breaks in their wave forms can be used as time markers for abrupt processes in the FAC signatures.

The occurrence of the AI described above should coincide with a brightening of an auroral arc due to additional acceleration of auroral electrons by the anomalous electric field. However, the delay of a few tens of seconds is possible, owing to the difference in the electron and MHD transit times. The model considered predicts an excitation of a very spatially localized response, because no compressional mode is generated during the anomalous resistance onset.
8.1 Estimates of critical plasma parameters and AI peak magnitudes

Let us estimate the expected parameters of the AI related to the ARL emergence. The threshold for the excitation of plasma instabilities and related anomalous resistance by the field-aligned current is different for different types of turbulence. It is highest for the Buneman instability $j^{*} \simeq e n u_{e} \simeq 5 \cdot 10^{-5} \mathrm{~A} / \mathrm{m}^{2}$, it is lower for the ion-sound instability $j^{*} \simeq e n u_{s} \simeq 10^{-5} \mathrm{~A} / \mathrm{m}^{2}$, and it is even lower for the ion-cyclotron instability $\sim 5 \cdot 10^{-6} \mathrm{~A} / \mathrm{m}^{2}$ (Kindell and Kennel, 1971). Thresholds values for all these instabilities have a deep and narrow minimum at altitudes $\sim 10^{3}-3 \cdot 10^{3} \mathrm{~km}$, where the formation of ARL could be expected. The ionospheric projection of the ARL is about the width of the auroral arc. The ARL width, as well as the auroral arc thickness, may vary over a wide range, from fractions of $\mathrm{km}$ to hundreds of $\mathrm{km}$.

The Alfvén damping scale $\lambda_{A 0}$ is mainly determined by a magnitude of the anomalous resistivity. However, estimates of $\sigma_{\|}$, being very uncertain, may vary in a wide range. Numerical modeling by Strelzov and Lotko (2003) of Alfvén wave interaction with the ARL demonstrated a significant wave reflection with transverse scales $\sim 20 \mathrm{~km}$. We suppose that this scale should coincide with $\lambda_{A 0}$.

The following order-of-magnitude estimates of possible peak values can be obtained from the above formulas for the east-west elongated ARL. The transverse radial electric field of the $\mathrm{AI}$ is $E \simeq E_{0} \simeq j_{0} \lambda_{A 0} \Sigma_{A}^{-1}$, and corresponding azimuthal magnetic component is $B \simeq\left(c / V_{A}\right) E_{0}=4 \pi j_{0} \lambda_{A 0} c^{-1}$. The drop in the potential across the ARL is $\{\varphi\} \simeq 2 \varphi_{0}=j_{0} Q$. The induced field-aligned current density is comparable to the initial global current density $j_{z A} \simeq j_{0}$. The disturbance of total current is $J \simeq 2 \Sigma_{A} E_{0}=2 j_{0} \lambda_{A 0}$. The ground disturbance has a bipolar latitudinal structure with a phase reversal just beneath the ARL ionospheric projection. If the ARL is wide, as compared with the ionospheric height $(a \geq h)$, the ionosphere does not produce substantial geometric attenuation of the magnetic impulse. However, even if the ARL is narrow $(a \ll h)$, in the case $\lambda_{A} \ll h$, this attenuation is not dramatically large, and the ground magnetic response could possibly be revealed from ground magnetograms. The peak ground signal is

$H^{(m)} \simeq H^{(g)}\left(\frac{\lambda_{A 0}}{h}\right)^{2}=\frac{8 \Sigma_{H}}{\Sigma_{A}+\Sigma_{P}} \frac{\lambda_{A 0}^{2} j_{0}}{c h}$.

These peculiarities may help to discriminate this impulse from the transient responses produced by other mechanisms, such as a burst of precipitating electrons, or cross-tail current disruption.

The AI related to the onset of anomalous conductivity may be masked by transient responses from other impulsive disturbances during the substorm break-up. Thus, at auroral field lines, where ARL occurs, the Pi2 waveforms must be rather complicated due to the superposition of transient signals from several impulsive sources. At some distance from 

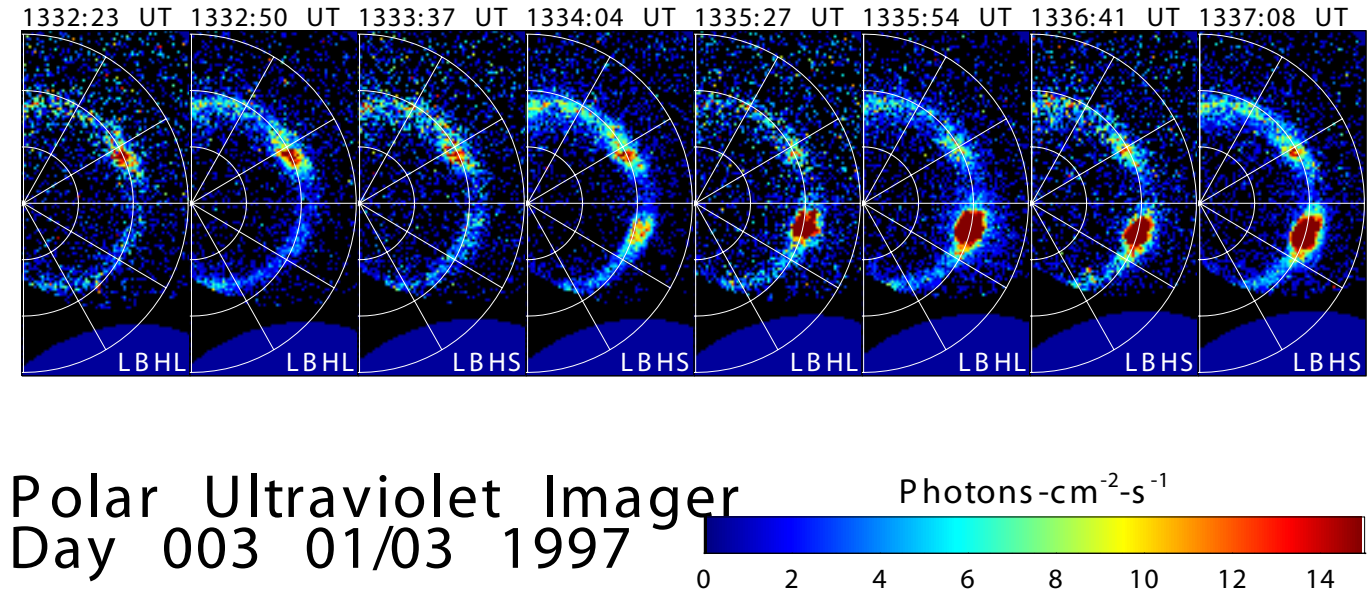

Fig. 10. The snapshots of UVI (LBHL) aurora made by Polar on 3 January 1997.

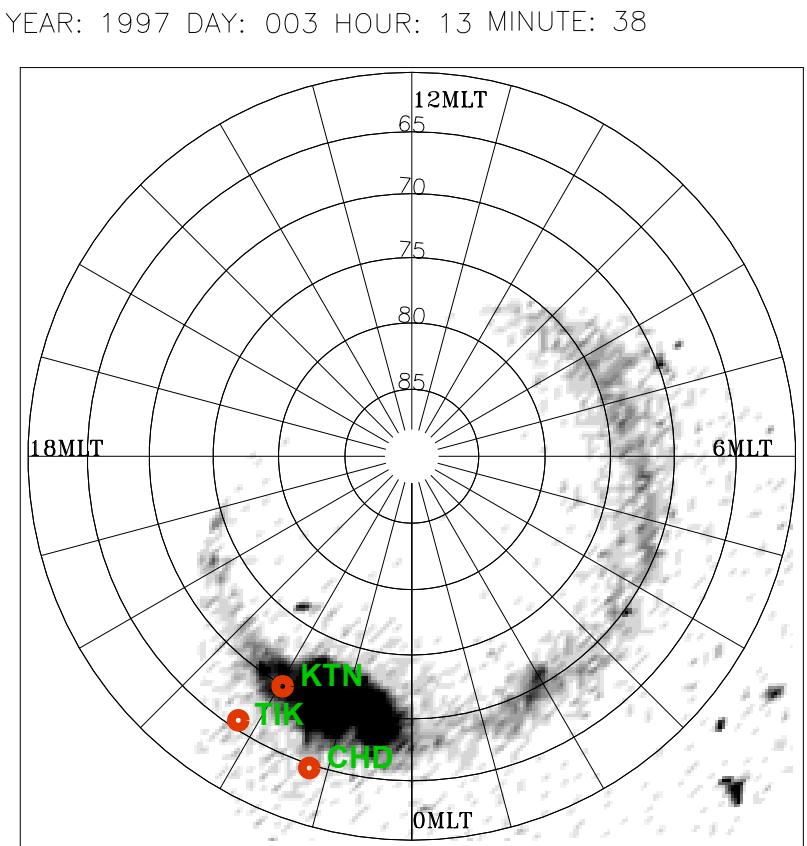

Fig. 11. The mapping of Polar UVI image onto the position of CPMN stations in Northern Russia at 3 January 1997, 13:38:22 UT (in geomagnetic coordinates).

these field lines, the disturbance caused by the excited AI decays fast, and more simple waveforms can be observed.

It is necessary to indicate that we have considered here the non-consistent problem - the onset of $\sigma_{\|}$under steady $j_{0}$, owing to fluctuations of plasma parameters near the threshold (that is, the external turn-on). To elucidate fully the physics of AI, an additional problem should be analyzed: a self-consistent description of the $\sigma_{\|}$onset by a time-increasing $j_{0}$. Elsewhere, we are going to consider this self-consistent problem.
A detailed consideration of the further evolution of the excited AI is beyond the scope of this paper. Transient AI will interact with the ionosphere and the ARLs in the Northern and Southern hemispheres. Upon this interaction parts of the incident wave energy are reflected and absorbed by ARL, and part penetrates through the ARL (Lysak and Dum, 1983; Trakhtenhertz and Feldstein, 1985). As a result, some part of the AI energy will be trapped in the Alfvén quasi-resonator between the conjugate ARLs, and part will be trapped in the cavity between the ionosphere and the ARL (Pilipenko et al., 2002; Streltsov and Lotko, 2003). If the reflection from the ARL does occur, the period of transient response to the excited Alfvén impulse will be shorter than the fundamental eigenperiod of the same field line.

\section{Event on 3 January 1997}

As a qualitative illustration of the principal feasibility of the considered effect, we consider the event on 3 January 1997. This substorm-like intensification with an amplitude of about $100 \mathrm{nT}$ has been detected by stations from the Circum-Pacific Magnetometer Network (CPMN) at 13:3013:50 UT (Fig. 9): Kotelny (KTN, geomagnetic coordinates: $69.9^{\circ}, 201.0^{\circ}, L=8.5, \mathrm{MLT} \simeq \mathrm{UT}+16$ ), Tixie (TIK, 65.7 , $196.9^{\circ}, L=5.9, \mathrm{MLT} \simeq \mathrm{UT}+16$ ), and Chokurdakh (CHD, $\left.64.8^{\circ}, 212.4^{\circ}, L=5.6, \mathrm{MLT} \simeq \mathrm{UT}+15\right)$. A decrease in the magnetic field $\mathrm{H}$ component started at 13:31 UT. However, at 13:34 UT the rate of the magnetic field decrease rapidly changed. The trend of the development of the bay is drawn as a dashed curve. This moment coincided with the auroral UVI intensification as observed by the Polar satellite (Fig. 10). Another rapid change of the magnetic field decrease and aurora intensification occurred at 13:37 UT.

These moments, 13:34 UT and 13:37 UT, of rapid change in the $\mathrm{H}$ component decrease rate and the auroral brightening were accompanied by the excitation of oscillatory transients. 
Band-pass filtering (40-100 s) indicates the occurrence of Pi2-like oscillations at KTN with a period of about $1 \mathrm{~min}$ and a peak-to-peak amplitude $\sim 10 \mathrm{nT}$.

The mapping of the Polar UV image, corresponding to the moment 13:38 UT just after the aurora intensification, onto the CPMN stations positions, shown in Fig. 11, indicates that only KTN was beneath the arc intensification region, whereas the stations TIX and CHD were beyond it, though not very far. As Fig. 9 shows, the Pi2-like signals were detected at KTN only, at TIK their amplitudes did not exceed $1 \mathrm{nT}$. Thus, the ground transient response is highly localized within the region of the aurora intensification.

At the recovery phase of this bay, Pc5 oscillations with a period $\sim 2.5-3.0 \mathrm{~min}$ were observed (Fig. 9). The period of these oscillations probably corresponds to the fundamental eigenperiod of the field line. Thus, the Pi2 period is shorter than the expected fundamental eigenperiod.

The basic features of this event agree with the scenario of a two-phase substorm development and the predictions of the AI generation model. However, the given example was intended to show the occurrence of localized Pi2-like response, and spatial and temporal correspondence between the magnetic transients and auroral activation, but it cannot be considered as firm evidence in favor of the suggested mechanism. The auroral bulge as seen in the Polar image may comprise many discrete auroral arcs, each with an individual time evolution and its magnetic fingerprint. To discriminate contributions to ground magnetic response from different source mechanisms during a substorm onset or auroral activation it is necessary to have a more dense (than CPMN) network of magnetic stations and auroral observations with a higher spatial resolution.

\section{Conclusion}

Following the idea of Arykov and Maltsev (1983), we have shown that the onset of anomalous resistance on auroral field lines is accompanied by the excitation of an AI. The generated $\mathrm{AI}$ indicates the transition of the global magnetosphereionosphere instability into an explosive phase with a positive feedback. The spatial structure of this impulse, both in space and on the ground, has been described both analytically and numerically under the thin layer approximation. The field-aligned current transported by the Alfvénic impulse is concentrated near the edges of the layer with anomalous resistivity, whereas the reverse currents are spread over the layer. According to our estimates, the AI amplitude may be sufficient to be detected by ground magnetometers under favorable conditions. Observations indicate that this mechanism may be responsible for some Pi2-like wave transients detected during an auroral activation.

Acknowledgements. The Polar data were provided by C. Meng and K. Liou from the Johns Hopkins University, Applied Physics Laboratory.

We appreciate the helpful comments of both reviewers. This research is supported by the fellowship from Heiwa Foundation (VA),
INTAS grant 03-05-5359 (NM), and RFBR grant 03-05-64670 (EF).

Topical Editor T. Pulkkinen thanks T. Bösinger and another referee for their help in evaluating this paper.

\section{References}

Arykov, A. A. and Maltsev, Yu. P.: Generation of Alfvén waves in an anomalous resistivity region. Planet. Space Sci., 31, 267-273, 1983.

Baumjohann, W. and Glassmeier, K. H.: The transient response mechanism and Pi2 pulsations at substorm onset - Review and outlook, Planet. Space Sci., 32, 1361-1370, 1984.

Baransky, L. N., Sterlikova, I. V., Troitskaya, V. A., Gokhberg, M. B., Pilipenko, V. A., and Munch, J.: Investigation of Pi2 pulsations along geomagnetic meridian, I. Meridional distribution of intensity and spectral content, Geomagn. Aeronomy, 20, 896904, 1980.

Belyaev, P. P., Bösinger, T., Isaev, S. V., and Kangas, J.: First evidence at high latitudes for the ionospheric Alfvén resonator, J. Geophys. Res., 104, 4305-4317, 1990.

Fedorov, E., Pilipenko, V., and Engebretson, M. J.: ULF wave damping in the auroral acceleration region, J. Geophys. Res., 106, 6203-6212, 2001.

Groot-Hedlin, C. D. and Rostoker, G.: Magnetic signatures of precursors to substorm expansive phase onset, J. Geophys. Res., 92, 5845-5856, 1987.

Kepko, L., and Kivelson, M. G.: Generation of Pi2 pulsations by bursty bulk flows, J. Geophys. Res., 104, 25 021-25 034, 1999.

Kepko, L., Kivelson, M. G., and Yumoto, K.: Flow bursts, braking, and Pi2 pulsations, J. Geophys. Res., 106, 1903-1915, 2001.

Kindel, J. M. and Kennel, C. F.: Topside current instabilities. J. Geophys. Res., 76, 3055, 1971.

Liou, K., Meng, C.-I., Lui, A. T. Y., Newell, P. T., Brittnacher, M., Parks, G., Reeves, G. D., Anderson, R. R., and Yumoto, K.: On relative timing in substorm onset signatures, J. Geophys. Res., 104, 22 807-22 817, 1999.

Liou, K., Meng, C.-I., Newell, P. T., Takahashi, K., Ohtani, S.-I., Lui, A. T. Y., Brittnacher, M., and Parks, G.: Evaluation of lowlatitude $\mathrm{Pi} 2$ pulsations as indicators of substorm onset using Polar ultraviolet imagery, J. Geophys. Res., 105, 2495-2505, 2000.

Lui, A. T. Y.,: Current disruption in the Earth's magnetosphere: Observations and models, J. Geophys. Res., 101, 1 067-13 088, 1996.

Lysak, R. L. and Dum, C. T.: Dynamics of magnetosphereionosphere coupling including turbulent transport, J. Geophys. Res., 88, 365-380, 1983.

Lysak, R. L. and Song, Y.: Energetics of the ionospheric feedback interaction, J. Geophys. Res., 107, 1160, doi:10.1029/2001JA000308, 2002.

Maltsev, Yu. P., Leont'ev, S. V., and Lyatsky, V. B.: Pi2 pulsations as a result of evolution of an Alfvén impulse originating in the ionosphere during a brightening of aurora, Planet. Space Sci. 22, 1519-1523, 1974.

Olson, J.V.: Pi2 pulsations and substorm onsets: A review, J. Geophys. Res., 104, 17 499-17 520, 1999.

Pilipenko, V., Fedorov, E., and Engebretson, M. J.: Alfvén resonator in the topside ionosphere beneath the auroral acceleration region, J. Geophys. Res., 107, SMP22 1-10, 2002.

Pokhotelov, O. A., Khruschev, V., Parrot, M., Senchenkov, S., and Pavlenko, V. P.: Ionospheric Alfvén resonator revisited: Feed- 
back instability, J. Geophys. Res., 106, 25 813-25 824, 2001.

Shiokawa, K., Baumjohann, W., Haerendel, G., et al.: High-speed ion flow, substorm current wedge, and multiple Pi2 pulsations, J. Geophys. Res., 103, 4491-4508, 1998.

Shiokawa, K., Yumoto, K., and Olson, J. V.: Multiple auroral brightenings and associated Pi2 pulsations, Geophys. Res. Lett., 29, doi:10.1029/2001GL014583, 2002.

Streltsov, A. V. and Lotko, W.: Reflection and absorption of Alfvénic power in the low-altitude magnetosphere, J. Geophys. Res., 108, 8016, doi:10.1029/2002JA009425, 2003.

Sutcliffe, P. R. and Yumoto, K.: On the cavity mode nature of lowlatitude Pi2 pulsations, J. Geophys. Res., 96, 1543-1551, 1991.

Trakhtenhertz, V. Yu. and Feldstein, A. Ya.: About dissipation of Alfvén waves in the layer with anomalous resistance, Geomag. Aeronomy, 25, 334-336, 1985.

Trakhtengertz, V. Yu. and Feldstein, A. Ya.: Auroral ionosphere electrodynamics during the switch-on of the energetic particles source, Geomag. Aeronomy, 28, 598-605, 1988.
Uozumi, T., Yumoto, K., Kawano, H., Yoshikawa, A., O1son, J. V., Solovyev, S. I., and Vershinin, E. F.: Characteristics of energy transfer of Pi2 magnetic pulsations: Latitudinal dependence, Geophys. Res. Lett., 27, 1619-1622, doi:10.1029/1999GL010767, 2000.

Uozumi T., Yumoto, K., Kawano, H., et al.: Propagation characteristics of Pi2 magnetic pulsations observed at ground high latitudes, J. Geophys. Res., 109, A08203, doi:10.1029/2003JA009898, 2004.

Vogt, J. and G. Haerendal: Reflection and transmission of Alfvén waves at the auroral acceleration region, Geophys. Res. Lett., 25, 277-280, 1998.

Yeoman, T. K., Milling, D. K., and Orr, D.: Polarization, propagation and MHD wave modes of Pi2 pulsations: SABRE/SAMNET results, Planet. Space Sci., 39, 983-998, 1991.

Yumoto, K., Takahashi, K., Sakurai, T., Sutcliffe, P. R., Kokubun, S., Luhr, H., Saito, T., Kuwashima, M., and Sato, N.: Multiple ground-based and satellite observations of global Pi2 magnetic pulsations, J. Geophys. Res., 95, 15 175-15 184, 1990. 